\title{
Estudo comparativo de três métodos de localização de escotomas centrais
}

\author{
Comparative study of three methods of central scotoma localization
}

\author{
Mylene Leal Matsuhara ${ }^{1}$ \\ Luciene Chaves Fernandes ${ }^{2}$
}

Trabalho realizado no serviço de Visão Subnormal do Hospital São Geraldo - Hospital das Clínicas da Universidade Federal de Minas Gerais - UFMG.

${ }^{1}$ Médica oftalmologista, coordenadora do serviço de Visão Subnormal do Instituto de Olhos de Belo Horizonte - IOBH.

${ }^{2}$ Coordenadora do serviço de Visão Subnormal do Hospital São Geraldo, Doutora em Oftalmologia pela Faculdade de Medicina da Universidade Federal de Minas Gerais - UFMG.

Endereço para correspondência: Mylene Leal Matsuhara Rua Clementino Viana Dotti, 121/ 202 - Belo Horizonte (MG) CEP 30575-130

E-mail: mylenemat@terra.com.br

Recebido para publicação em 30.01.2003

Versão revisada recebida em 30.06.2003

Aprovação em 15.08.2003

\begin{tabular}{|l|}
\hline RESUMO \\
\hline Objetivo: O objetivo do presente estudo foi comparar a sensibilidade de \\
três métodos de localização de escotomas centrais. Métodos: Foram estu- \\
dados 29 pacientes, com idade variando entre 10 e 78 anos, encaminhados \\
ao Serviço de Visão Subnormal do Hospital São Geraldo da Faculdade de \\
Medicina da UFMG, com diagnóstico prévio de doenças com escotoma \\
central. Foram utilizados os métodos carta de Asmler, observação da face \\
e observação da tabela de leitura para perto. Resultados: Dentre estes \\
métodos, o que obteve o melhor desempenho, ou seja, apresentou maior \\
percentual de pacientes nos quais se constatou a presença de escotomas, \\
foi a carta de Asmler (63,6\%), seguida pela observação da face (47,3\%) e \\
observação da tabela de leitura para perto (30,9\%) (p<0,05). Todos os três \\
métodos de estudo apresentaram baixa sensibilidade, possivelmente \\
explicada pela plasticidade do sistema visual em tentar compensar a área \\
escotomatosa. Conclusão: O presente resultado demonstra a necessidade \\
da aplicação de novas técnicas que melhorem a sensibilidade dos métodos \\
atualmente disponíveis para localização dos escotomas centrais.
\end{tabular}

Descritores: Escotoma; Baixa visão; Observação/métodos; Testes visuais/métodos

\section{INTRODUÇÃO}

Escotoma pode ser definido como uma área não responsiva a estímulo luminoso específico dentro de um campo visual normal. Patologias retinianas com escotomas centrais de diversas etiologias constituem a principal causa de baixa acuidade visual não refrativa ${ }^{(1)}$. A presença do escotoma central leva a necessidade de técnicas de reabilitação visual. Entre tais técnicas a utilização de magnificação e controle da luminosidade tem se mostrado ineficientes para alguns pacientes ${ }^{(2)}$. Tais pacientes se beneficiariam da utilização da visão excêntrica. Visão excêntrica significa a utilização de uma área não foveal, que pode ser alcançada espontaneamente ou através de treinamento ${ }^{(2)}$. O primeiro passo para um treinamento eficiente é conscientizar o paciente da presença do escotoma ${ }^{(3)}$.

A carta de Amsler é usada como método de localização e monitorização de escotomas nos 10 graus centrais, monitorização de metamorfopsia, bem como avaliação grosseira da percepção de contraste desde $1940^{(4-5)}$. Por ser um método de rápida execução, fácil interpretação e baixo custo tornou-se amplamente difundido no meio oftalmológico.

O'Connell cita dois outros métodos de localização dos escotomas: observação da face e observação da tabela de leitura para perto ${ }^{(3)}$. No primeiro, quando o paciente não evidencia estruturas da face do examinador pensa-se na presença de escotoma. No segundo, a falha na leitura de letras na linha testada também evidencia a presença do escotoma. 
O presente trabalho compara três métodos de localização do escotoma central com o intuito de observar sua sensibilidade.

\section{MÉTODOS}

\section{Casuística}

Pacientes do serviço de visão subnormal do Hospital São Geraldo maiores de 10 anos, acuidade visual >20/400, encaminhados ao serviço com diagnóstico de doenças com acometimento do campo visual central.

\section{Método}

Os pacientes que preencheram os pré-requisitos acima foram submetidos a uma avaliação especializada que incluía anamnese, medida atualizada da acuidade visual longe e perto através das tabelas LH (Lea Hyvärinen) para iletrados e ETDRS para letrados em uso da melhor correção visual. Os valores foram anotados em pés. Aplicou-se então os testes de pesquisa do escotoma central.

\section{Métodos de pesquisa do escotoma}

3.1. Observação da face: Com o paciente utilizando sua melhor correção para longe, e a uma distância de 1 metro do examinador, foi solicitado que observasse monocularmente a face do examinador devidamente iluminada com um foco de luz incandescente de 60 watts, mantendo a fixação no nariz do examinador. Questionou-se por área de borramento na face, ou seja, não percepção de estruturas como, por exemplo, olho esquerdo, nariz ou boca.

3.2. Observação da tabela de visão de perto: Utilizou-se a tabela de acuidade visual para perto devidamente iluminada e na distância preconizada de $40 \mathrm{~cm}$. Solicitouse ao paciente que localizasse e lesse a linha correspondente ao dobro de sua acuidade visual (ex $\mathrm{AV}=4 \mathrm{M}$ ler linha $8 \mathrm{M}$ ) em uso da sua melhor correção visual para longe acrescido de $+2,50$ no caso de paciente présbita. $\mathrm{O}$ teste foi considerado alterado quando o paciente não percebia pelo menos uma letra da linha em questão.

3.3. Carta de Amsler: Posicionou-se a carta de Amsler a uma distância de $33 \mathrm{~cm}$ do paciente utilizando sua correção visual para longe. Quando o paciente era présbita utilizava-se adição de $+3,00$ dioptrias. Iniciou-se o teste questionando ao mesmo se havia percepção do ponto central de fixação da prancha \#1. Caso a resposta fosse positiva solicitou-se ao mesmo que delimitasse a área de distorção e fez-se anotação conforme padronização (vermelho - olho direito, verde olho esquerdo). Caso a resposta fosse negativa utilizou-se a prancha \# 2 que apresenta um x para auxiliar o paciente a fixar a área central. Deu-se sequiência questionando por áreas de distorção e fazendo anotação conforme descrito acima. A figura 1 exemplifica a anotação do teste da carta de Amsler.

3.4. Estudo estatístico: O índice de concordância de Kappa é um teste não-paramétrico utilizado para avaliar o nível de concordância entre exames ${ }^{(6)}$. No trabalho, o índice de Kappa forneceu o nível de concordância entre os resultados dos três métodos aqui estudados. O índice de Kappa foi interpretado conforme demonstrado no quadro 1.

Com o intuito de avaliar qual dos três métodos de localização de escotoma apresentava o melhor resultado utilizou-se o teste de Cochran. Este teste tem como objetivo detectar a presença ou não de diferenças entre os três métodos aqui estudados. O teste de Cochran é utilizado quando as respostas do tratamento são do tipo "Sucesso" ou "Insucesso", "Alterado" ou "Não alterado" e, além disso, cada unidade experimental é avaliada em relação a mais de dois tratamentos distintos. Todos os resultados foram considerados significativos a um nível de significância de $5 \%$ ( $\mathrm{p}<0,05)$, tendo, portanto, $95 \%$ de confiança de que os resultados estejam corretos.

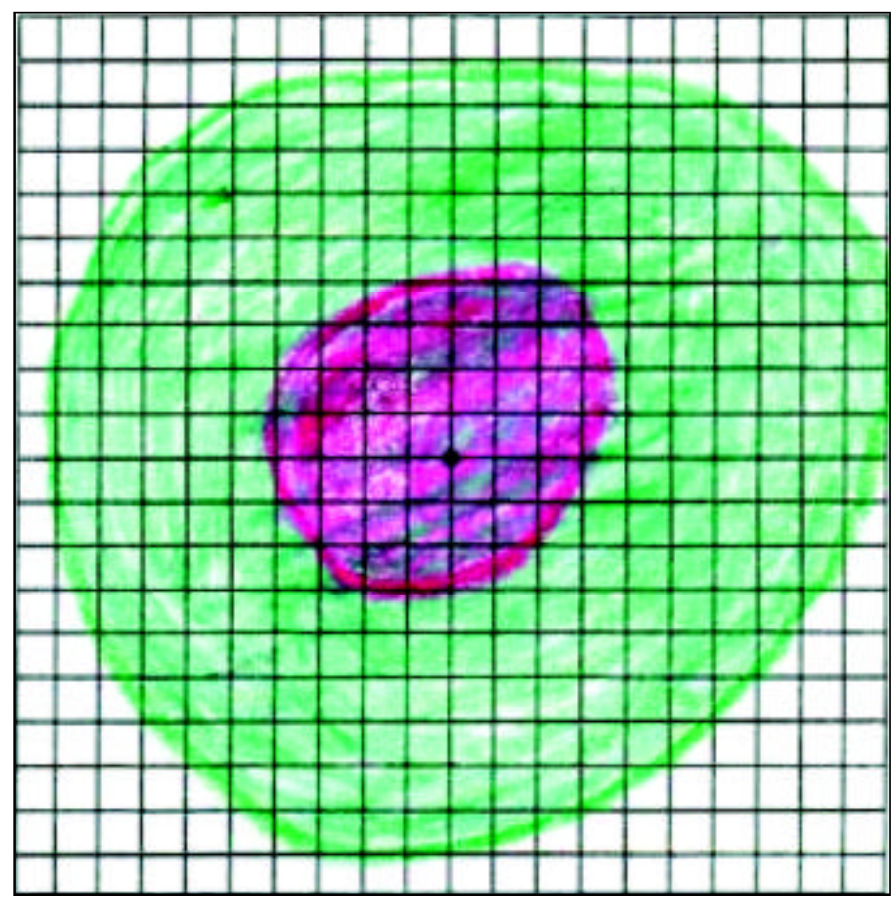

Figura 1 - Anotação do teste da carta de Amsler

Quadro 1. Interpretação da estatística "Índice Kappa"

\begin{tabular}{ll} 
Intervalo & Interpretação \\
$<0,40$ & Concordância fraca \\
$0,40 \vdash 0,75$ & Concordância de razoável à boa \\
$\geq 0,75$ & Concordância excelente \\
\hline
\end{tabular}




\section{RESULTADOS}

Os dados a seguir referem-se ao estudo realizado em 29 pacientes (55 olhos) sabidamente portadores de patologias com escotoma central. A tabela 1 mostra que a idade dos pacientes variou entre 10 a 78 anos com média aproximada de 40 anos. Já a tabela 2 mostra uma caracterização dos pacientes em relação à faixa etária. A tabela 3 mostra uma distribuição dos pacientes quanto ao diagnóstico apresentado.

As tabelas 4 a 6 mostram que existe concordância significativa $(p<0,05)$ entre os 3 exames avaliados tomados 2 a 2. E, apesar da concordância ser significativa, o nível de concordância entre os exames Observação da face e Observação da tabela de perto é considerado de razoável a bom (Tabela 4) e para as demais avaliações o nível de concordância é fraco (Tabelas 5 e 6).

Pode-se observar pela tabela 7 que existe diferença significativa entre os três métodos, ou seja, os três métodos avaliam de forma diferente o percentual de resultados com alteração. Sendo que, a Carta de Amsler foi o método que diagnosticou o maior percentual de exames com alteração seguida pelo método Observação da face e por último, o pior deles, o método observação da tabela de perto.

Já o gráfico 1 mostra que 72,7\% (40 casos) dos exames apresentaram alterações quando se associou os três métodos de detecção de escotomas. Portanto, além dos 35 casos diagnosticados pelo método Carta de Amsler, apenas cinco casos foram confirmados após a utilização dos métodos observação da face e observação da tabela de perto.

\section{DISCUSSÃO}

Pessoas com escotoma nos seus 10 graus centrais de visão podem não noticiar estes pontos cegos em seu campo

Tabela 1. Análise descritiva da idade dos pacientes submetidos ao estudo de escotomas centrais

\begin{tabular}{|lccccc|} 
Variável & N & \multicolumn{4}{c}{ Medidas descritivas } \\
\cline { 3 - 6 } Idade (anos) & 29 & 10,0 & 78,0 & 39,9 & 23,0 \\
\hline
\end{tabular}

\begin{tabular}{|c|c|c|}
\hline \multirow{2}{*}{ Faixa etária } & \multicolumn{2}{|c|}{ Freqüência } \\
\hline & $\mathbf{N}$ & $\%$ \\
\hline De 10 a 14 anos & 4 & 13,8 \\
\hline De 15 a 20 anos & 5 & 17,2 \\
\hline De 21 a 30 anos & 2 & 6,9 \\
\hline De 31 a 40 anos & 7 & 24,2 \\
\hline De 41 a 50 anos & 1 & 3,5 \\
\hline De 51 a 60 anos & 2 & 6,9 \\
\hline De 61 a 70 anos & 3 & 10,3 \\
\hline De 71 a 80 anos & 5 & 17,2 \\
\hline Total & 29 & 100,0 \\
\hline
\end{tabular}

Tabela 3. Distribuição dos pacientes submetidos à pesquisa do escotoma central quanto ao diagnóstico

\begin{tabular}{lrr} 
Diagnóstico & \multicolumn{2}{c}{ Freqüência } \\
\cline { 2 - 3 } & N & $\%$ \\
Stargardt & 4 & 13,8 \\
DMRI $^{*}$ & 5 & 17,2 \\
Uveíte por TBC ** & 2 & 7,0 \\
Neuropatia a esclarecer & 2 & 6,9 \\
Toxoplasmose & 11 & 37,9 \\
Atrofia óptica & 1 & 3,5 \\
Distrofia de cones & 1 & 3,5 \\
Maculopatia de Best & 1 & 3,5 \\
Estrias angióides & 2 & 6,9 \\
Buraco macular & 1 & 3,5
\end{tabular}

Nota: As porcentagens na tabela somam mais de $100 \%$, pois, cada paciente poderia apresentar mais de um diagnóstico. Cada porcentagem foi calculada em relação ao total de 29 pacientes.

*DMRI: degeneração macular relacionada à idade; **TBC: tuberculose

\begin{tabular}{|c|c|c|c|c|c|}
\hline \multirow{3}{*}{$\begin{array}{l}\text { Observação } \\
\text { da face }\end{array}$} & \multicolumn{4}{|c|}{ Observação da tabela de perto } & \multirow{3}{*}{ Total } \\
\hline & \multicolumn{2}{|c|}{ Sem alteração } & \multicolumn{2}{|c|}{ Com alteração } & \\
\hline & $\mathbf{N}$ & $\%$ & $\mathbf{N}$ & $\%$ & \\
\hline Sem alteração & 27 & 49,1 & 2 & 3,6 & 29 \\
\hline Com alteração & 11 & 20,0 & 15 & 27,3 & 26 \\
\hline Total & 38 & & 17 & & 55 \\
\hline
\end{tabular}

Tabela 5. Análise de concordância entre os exames observação da face e carta de Amsler

\begin{tabular}{|c|c|c|c|c|c|}
\hline \multirow{3}{*}{$\begin{array}{l}\text { Observação } \\
\text { da face }\end{array}$} & \multicolumn{4}{|c|}{ Carta de Amsler } & \multirow{3}{*}{ Total } \\
\hline & \multicolumn{2}{|c|}{ Sem alteração } & \multicolumn{2}{|c|}{ Com alteração } & \\
\hline & $\mathbf{N}$ & $\%$ & $\mathbf{N}$ & $\%$ & \\
\hline Sem alteração & 16 & 29,1 & 13 & 23,6 & 29 \\
\hline Com alteração & 4 & 7,3 & 22 & 40,0 & 26 \\
\hline Total & 20 & & 35 & & 55 \\
\hline
\end{tabular}

Nota: Kappa $=0,39 ; p=0,0044$.

Porcentagens em relação ao número total geral de olhos apresentado na tabela

Tabela 6. Análise de concordância entre os exames observação da tabela de perto e carta de Amsler

\begin{tabular}{|c|c|c|c|c|c|}
\hline \multirow{3}{*}{$\begin{array}{l}\text { Observação da } \\
\text { tabela de perto }\end{array}$} & \multicolumn{4}{|c|}{ Carta de Amsler } & \multirow{3}{*}{ Total } \\
\hline & \multicolumn{2}{|c|}{ Sem alteração } & \multicolumn{2}{|c|}{ Com alteração } & \\
\hline & $\mathbf{N}$ & $\%$ & $\mathbf{N}$ & $\%$ & \\
\hline Sem alteração & 18 & 32,7 & 20 & 36,4 & 38 \\
\hline Com alteração & 2 & 3,6 & 15 & 27,3 & 17 \\
\hline Total & 20 & & 35 & & 55 \\
\hline
\end{tabular}




\begin{tabular}{|c|c|c|c|c|c|}
\hline \multirow{3}{*}{ Método } & \multicolumn{4}{|c|}{ Resultado do exame } & \multirow{3}{*}{ Total } \\
\hline & \multicolumn{2}{|c|}{ Sem alteração } & \multicolumn{2}{|c|}{ Com alteração } & \\
\hline & $\mathbf{N}$ & $\%$ & $\mathbf{N}$ & $\%$ & \\
\hline $\begin{array}{l}\text { Observação } \\
\text { da face }\end{array}$ & 29 & 52,7 & 26 & 47,3 & 55 \\
\hline $\begin{array}{l}\text { Observação da } \\
\text { tabela de perto }\end{array}$ & 38 & 69,1 & 17 & 30,9 & 55 \\
\hline Carta de Amsler & 20 & 36,4 & 35 & 63,6 & 55 \\
\hline
\end{tabular}

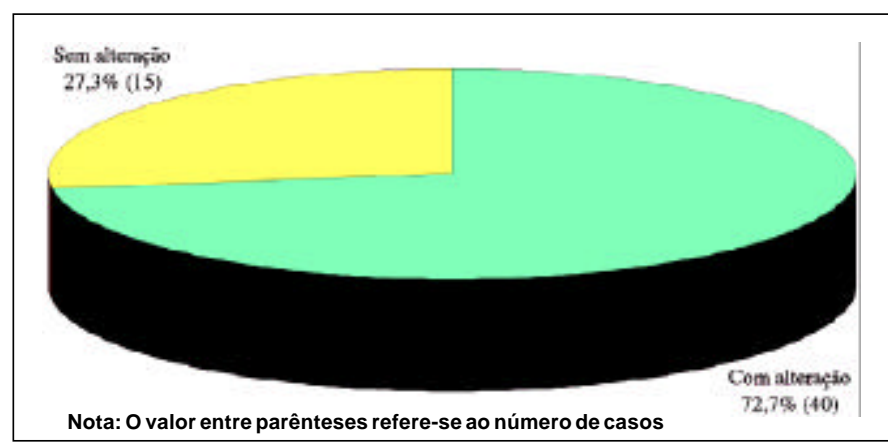

Gráfico 1 - Percentual de exames alterados após a associação dos três métodos de localização de escotoma

de visão e terem dificuldades na "performance" visual que supera a esperada pelos testes de acuidade padrões. Esta não percepção pode ser resultante de uma tentativa do sistema visual de se adaptar ao escotoma central, tais quais o desenvolvimento do ponto retiniano preferencial e o fenômeno de complementação percentual ${ }^{(1,7)}$. Baixa sensibilidade e variação intra-individual encontrada nos testes de Amsler são em parte resultantes do fenômeno de complementação percentual ${ }^{(7-8)}$. Este fenômeno é dinâmico e um processo flutuante, assim dois testes consecutivos de Amsler no mesmo paciente não são idênticos, mesmo quando realizados pelo mesmo examinador e em intervalo de apenas 15 minutos $^{(7)}$.

Schuchard (1993) avaliou pacientes submetidos ao teste da carta de Amsler com o "scanning laser" oftalmoscópio, considerado atualmente o melhor método diagnóstico de escotoma, e constatou que a sensibilidade do teste carta de Amsler era muito baixa. Em seu estudo o teste falhou em detectar quase que $50 \%$ dos escotomas. E mesmo quando os escotomas eram detectados, a sua completa extensão não era notada ${ }^{(5)}$.

Não foi encontrado na literatura pesquisada nenhum trabalho com referência a resposta dos testes observação da face e observação da tabela de perto.

Conclui-se então que até o momento, descartando o "scanning laser" oftalmoscópico que ainda constitui método propedêutico inviável devido ao custo, não se dispõe de métodos clínicos diagnósticos com confiabilidade aceitável. Dos métodos estudados, o que apresentou melhor reprodutibilidade foi a carta de Asmler, mesmo assim com uma margem de erro de até $50 \%$.

A literatura cita técnicas que aumentariam a eficácia da carta de Asmler. Tais quais a permissão de observação binocular da grade de teste antes do exame monocular, a utilização de iluminação limiar com lentes polarizadas e a utilização de grades de testes vermelhas ${ }^{(3-5,9-10)}$.

\section{ABSTRACT}

Objective: The aim of this study was to compare the sensitivity of three methods of central scotoma localization. Methods: Twenty-nine patients (range 10-78 years) were referred to the Service of Subnormal Vision of the São Geraldo Hospital (Minas Gerais Federal University, School of Medicine) with previous diagnosis of diseases with central scotoma. The Asmler grid, face observation and near acuity test observation methods were used. Results: Among these methods, the one which presented the highest percentile of patients that reported scotoma presence was the Asmler grid (63.6\%), followed by face observation ( $47.3 \%)$ and near acuity test observation $(30.9 \%)(\mathrm{p}<0.05)$. The three methods presented low sensitivity, possibly explained by the plasticity of the visual system trying to compensate the scotoma area. Conclusion: The present result demonstrates that new techniques should be developed to improve the sensitivity of scotoma localization methods.

Keywords: Scotoma; Vision, low; Observation/methods; Vision tests/methods

\section{REFERÊNCIAS}

1. Faye EE. Clinical low vision. $2^{\text {nd }}$ ed. Boston: Little, Brow1984; p.529.

2. Goodrich GL, Mehr EB. Eccentric viewing training and low vision aids: Current practice and implications of peripheral retinal research. Am J Optom Physiol Opt 1986;63:119-26.

3. Cole RG, Rosenthal BP. Remediation and management of low vision. St. Louis: Mosby-Year Book 1996. p.296.

4. Achard OA, Safran AB, Duret FC, Ragama E. Role of the completion phenomenon in the evaluation of Amsler grid results. Am J Ophthalmol 1995; 120:322-9.

5. Schuchard RA. Validity and interpretation of Amsler grid reports. Arch Ophthalmol 1993;111:776-80.

6. Johnson RA, Bhattacharya GK. Statistics: principles and methods. New York: John Wiley; 1986. p.578.

7. Fletcher DC, Schuchard RA. Scanning laser ophthalmoscope macular perimetry and applications for low vision rehabilitation clinicians. Ophthalmol Clin North Am 1994; 7:257-65.

8. Schuchard RA. Adaptation to macular scotomas in persons with low vision. Am J Occup Ther 1995;49:870-6.

9. Wall M, Collins C, May DR. Low-intensity grids improve sensitivity of Amsler grid testing in diabetic patients without background retinopathy. Ann Ophthalmol 1990;22:96-100.

10. Mutlukan E, Cullen JF. Red colour comparison perimetry chart in neuro-opthalmological examination. Eye 1991;5:352-61. 